\title{
On the Optimal Shape of Magnetic Swimmers
}

\author{
Hermes Gadêlha* \\ Department of Applied Mathematics and Theoretical Physics, Centre for Mathematical Sciences, \\ University of Cambridge, Wilberforce Road, Cambridge CB3 0WA, United Kingdom \\ Received October 9, 2012; accepted January 25, 2013
}

\begin{abstract}
Magnetic actuation of elasto-magnetic devices has long been proposed as a simple way to propel fluid and achieve locomotion in environments dominated by viscous forces. Under the action of an oscillating magnetic field, a permanent magnet, when attached to an elastic tail, is able to generate bending waves and sufficient thrust for propulsion. We study the hydrodynamical effects of the magnetic head geometry using a geometrically exact formulation for the elastic tail elastohydrodynamics. We show that the spherical head geometry fails to take full advantage of the propulsive potential from the flexible tail. Nevertheless, while elongated prolate spheroids demonstrate a superior swimming performance, this is still regulated by the nature of the imposed magnetic field. Interestingly, the highest swimming speed is observed when the magnitude of the magnetic field is weak due to delays between the orientation of the magnetic moment and the oscillating magnetic field. This allows the stored elastic energy from the deformed tail to relax during the phase lag between the imposed magnetic field and the swimmer's magnetic moment, favouring in this way the net propulsion. In particular, this result suggests the existence of optimal magnetic actuations that are non-smooth, and even discontinuous in time, in order to fully explore the propulsive potential associated with the relaxation dynamics of periodically deformed elastic filaments.
\end{abstract}

MSC2010 numbers: 76Z99, 35Q35, 35Q74, 35Q93

DOI: $10.1134 / \mathrm{S} 156035471301005 \mathrm{X}$

Keywords: micro-swimmers, magnetic actuation, elastohydrodynamics and elastic filaments

\section{INTRODUCTION}

External actuation of artificial devices via the action of a magnetic field has long been employed for diverse applications in science and technology [1]. By constructively controlling magnetic objects, it is possible to perform mechanical work with great precision and without the need of a wired connection. At the micro-scale, it offers an elegant and non-invasive method to drive fluid and even induce locomotion in wireless magnetic devices $[3,5,6,17,26]$. Furthermore, due to the malleability of different magnetic materials, the versatility of this method has proven to be limitless and ranges from biomimetic magnetic devices, such as artificial cilia [10, 18], bacteria [13, 14] and spermatozoa [17, 22, 27], to fluid mixers [4], surface walkers [28] and micro-robots that are capable of carrying cargos at microscope precision [9, 24]. Nevertheless, the small-scale involved in these systems entails that the fluid viscous dissipation is dominant, suppressing in this way hydrodynamic inertial effects notorious for imposing a time-reversibility constraint [25] that is responsible for additional challenges on the design of micro-swimmer devices. In this regime, when the motion is reversed over time, each material point in the fluid retraces its history, implying that no timereversible motion can result in swimming at low Reynolds number, also known as the Purcell's scallop theorem [25]. It follows from the scallop constraint that low Reynolds number swimmers need to undergo a non-reciprocal motion in order to generate propulsion. Interestingly enough, traveling waves propagating down in flexible filaments [21, 29-31], such as the sperm flagellum [7,8], are non-reciprocal in time and this mechanism is a very common method of propulsion over a wide range of Reynolds numbers.

${ }^{*}$ E-mail: habg2@cam.ac.uk 


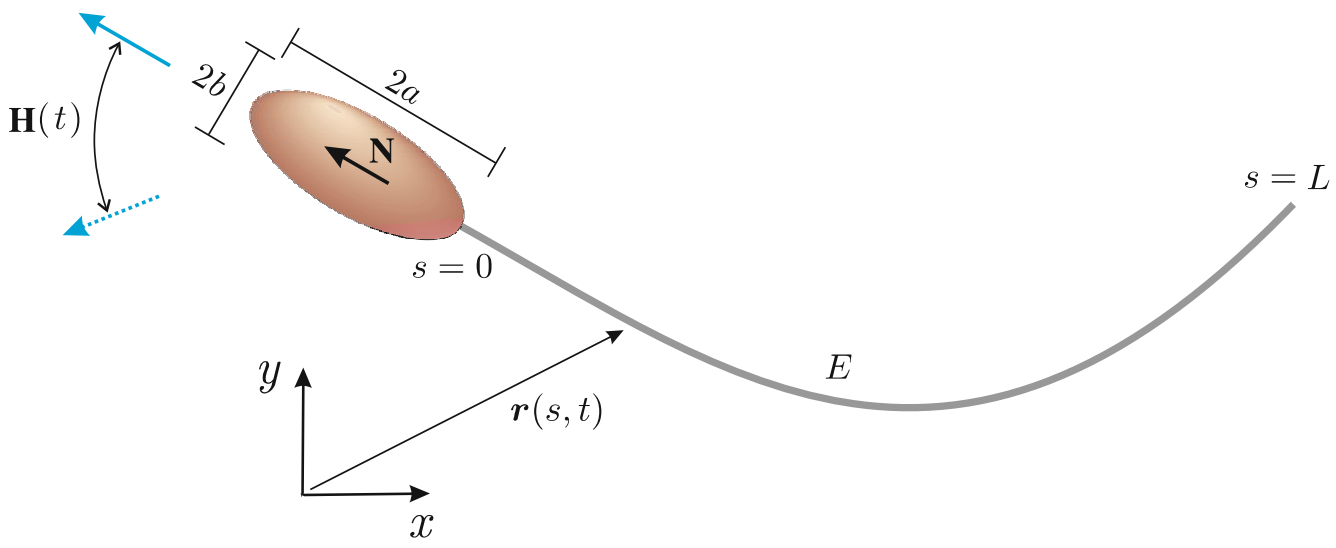

Fig. 1. (Colour online) Schematic of the elasto-magnetic swimmer composed of a prolate spheroidal permanent magnet and an elastic tail.

One of the simplest designs for a magnetic microswimmer, and yet highly effective while circumventing the time-reversibility constraint, consists of a permanent magnet head attached to a non-magnetic flexible tail [9, 22, 24, 27], as illustrated in Fig. 1. By imposing an oscillating magnetic field, the magnetic head forces the flexible tail to wiggle around in a non-reciprocal manner, ultimately resulting in the net propulsion of the magnetic swimmer. Here, we consider the hydrodynamic effect of different head geometries on the swimming behavior of such elastomagnetic devices, while coupling geometrically non-linear interactions arising from the flexible filament elastohydrodynamics. The resulting system is solved numerically, and the swimming speed is contrasted for different configurations of the magnetic field.

\section{GOVERNING EQUATIONS}

We consider prolate spheroidal, permanent magnets with an average magnetic moment $\mathbf{N}$, lying along the spheroid's polar axis, of length $2 a$ and equatorial radius $b$, as illustrated in Fig. 1 . The spheroidal magnetic head is rigidly anchored to a flexible, but inextensible, Euler-Bernoulli filament of bending stiffness $E$ and total length $L$, so that the tangent direction of the elastic filament coincides with the polar axis of the magnetic head at the point of attachment. The resulting magnetic swimmer is externally actuated by a periodic magnetic field $\mathbf{H}(t)$, constrained to the plane, while immersed in an inertialess viscous medium, characterized by low Reynolds number hydrodynamics. The bending wave generation along the filament, ultimately responsible for the propulsion of the swimmer, is therefore achieved via the magneto-elastic coupling between the flexible tail and the magnetic torque. The elastic tail is described by its neutral line $\boldsymbol{r}(s, t)$, relative to a fixed frame of reference, and parameterized by the arclength $s \in[0,1]$ and time $t$, nondimensionalized, respectively, by the length scale $L$ and time scale given by the frequency of oscillation of the magnetic field $\omega$. The flexible tail is free from body couples, and the total contact force $\mathbf{F}$ is implicitly specified by the total balance of angular momentum on the elastic slenderbody [20]. The motion of the elastic tail is dominated by the hydrodynamic drag experienced by each infinitesimal element along the filament [23, 30], simplified to leading order of the slender-body hydrodynamics [12] and nondimensionalized by $E / L^{2}$,

$$
\mathcal{L}^{4} \boldsymbol{r}_{t}=-\left[\mathbf{1}+(\gamma-1) \boldsymbol{r}_{s} \boldsymbol{r}_{s}\right] \cdot \boldsymbol{r}_{s s s s}+\tau \boldsymbol{r}_{s s}+\gamma \tau_{s} \boldsymbol{r}_{s}
$$

where $\boldsymbol{r}_{s} \equiv \partial \boldsymbol{r} / \partial s$ is the tangent vector to the centerline of the elastic tail, and the subscript $t$ denotes differentiation with respect to the dimensionless time. $\gamma=\xi_{\perp} / \xi_{\|}$represents the ratio between the perpendicular, $\xi_{\perp}$, and parallel, $\xi_{\|}$, fluid dynamic resistance coefficients, and is typically 2 for slender circular cylinders in infinite fluids. The dimensionless parameter

$$
\mathcal{L}=L\left(\frac{\xi_{\perp} \omega}{E}\right)^{1 / 4}
$$


elsewhere known as the sperm-compliance number [8], characterizes the relative importance of elastic forces to viscous drag $[23,30]$. The line tension $\tau(s, t)$ is the Lagrange multiplier determined by the filament inextensibility constraint $\boldsymbol{r}_{s} \cdot \boldsymbol{r}_{s}=1$. In Eq. (2.1) the time derivative and the anisotropic operator, described by the bracket term on the left-hand side, originate from the force-velocity linearity of the hydrodynamic coupling [12], while the fourth-order derivative in arclength and the line tension terms arise from the monotonicity between moment and curvature derived from the Euler-Bernoulli rod theory [20, 23, 30]. Equations of motion (2.1)-(2.2) describe the elastohydrodynamics of a passive elastic filament in a viscous fluid, and have been studied extensively in the literature $[7,11,16,23,30,31]$.

The contribution of the magnetic torque to the dynamics of the flexible swimmer is included by specifying the boundary conditions. At the right boundary, $s=1$, the elastic tail is free from external forces and torques, hence $\left.\left(-\boldsymbol{r}_{s s s}+\tau \boldsymbol{r}_{s}\right)\right|_{s=1}=0$ and $\left.\boldsymbol{r}_{s s}\right|_{s=1}=0$. At the point of attachment between the magnetic head and the flexible tail, $s=0$, the elastic filament experiences the viscous drag force, $\mathbf{F}_{\text {head }}$, and moment, $\mathbf{M}_{\text {head }}$, due to translations and rotations of the prolate spheroidal head in the fluid,

$$
\left(\begin{array}{c}
\mathbf{F}_{\text {head }} \\
\mathbf{M}_{\text {head }}
\end{array}\right)=\overline{\mathcal{L}}^{4} \mathcal{R}(a / L, t)\left(\begin{array}{c}
\mathbf{U} \\
\mathbf{\Omega}
\end{array}\right),
$$

where $\overline{\mathcal{L}}=L(\eta \omega / E)^{1 / 4}$, for a fluid viscosity $\eta$. $\mathcal{R}$ is the dimensionless grand resistance matrix of the magnetic head, given by analytical functions of the prolate spheroid's eccentricity $\epsilon=\sqrt{1-(1 / \ell)^{2}}$ and polar axis $2 a$, where we have defined $\ell=a / b[19]$. The torque and force balance at $s=0$ yields the required boundary condition for the magnetic swimmer in terms of the head velocity field $(\mathbf{U}, \boldsymbol{\Omega})$, as well as the imposed magnetic torque,

$$
\mathbf{T}_{\mathrm{H}}=m \boldsymbol{r}_{s} \times \hat{\boldsymbol{h}}
$$

via

$$
\begin{aligned}
\mathbf{F}_{\text {head }} & =\boldsymbol{r}_{s s s}-\tau \boldsymbol{r}_{s}, \\
\mathbf{M}_{\text {head }} & =\mathbf{T}_{\mathrm{H}}-\boldsymbol{r}_{s} \times \boldsymbol{r}_{s s},
\end{aligned}
$$

where $\hat{\boldsymbol{h}}$ is the dimensionless, normalized magnetic field. The magnetic number

$$
m=\frac{\mu_{0} v \mathrm{~N}_{0} \mathrm{H}_{0} L}{E}
$$

represents the ratio between the magnetic and elastic moments, given in terms of the permeability of free space $\mu_{0}$, the volume of the magnetic head $v$, and the strength of both magnetic field $\mathrm{H}_{0}$ and magnetization $\mathrm{N}_{0}$ of the permanent magnet [15]. A general discussion on different types of magnetic materials, the shape influence of the magnet, as well as their applications, is given in Ref. [1], and references therein.

\section{NUMERICAL SOLUTIONS}

Numerical solutions for the elasto-magnetic swimmer described by Eqs. (2.1)-(2.5) have been found by employing the numerical scheme devised in Gadêlha et al. [7] for the dynamics of sperm motility, which uses a combination of second-order finite differences and second-order implicit timestepping. The latter, in particular, has been validated against analytical and non-linear numerical solutions, in addition to experiments, for driven passive elastic filaments in viscous fluids [7, 31]. In order to study the influence of the magnetic head geometry on the overall behavior of the magnetic swimmer, we have considered four distinct prolate spheroidal shapes for the head by varying the head slenderness parameter $\ell=a / b$ from a simple spherical head, $\ell=1$, to an elongated prolate spheroid, $\ell=6$, with the same head volume and equivalent to a sphere of radius 0.05 . We allowed the applied magnetic field $\hat{\boldsymbol{h}}(t)$ to periodically vary its angular orientation relative to the fixed frame of reference,

$$
\phi(t)=\phi_{0} \sin t+\pi,
$$




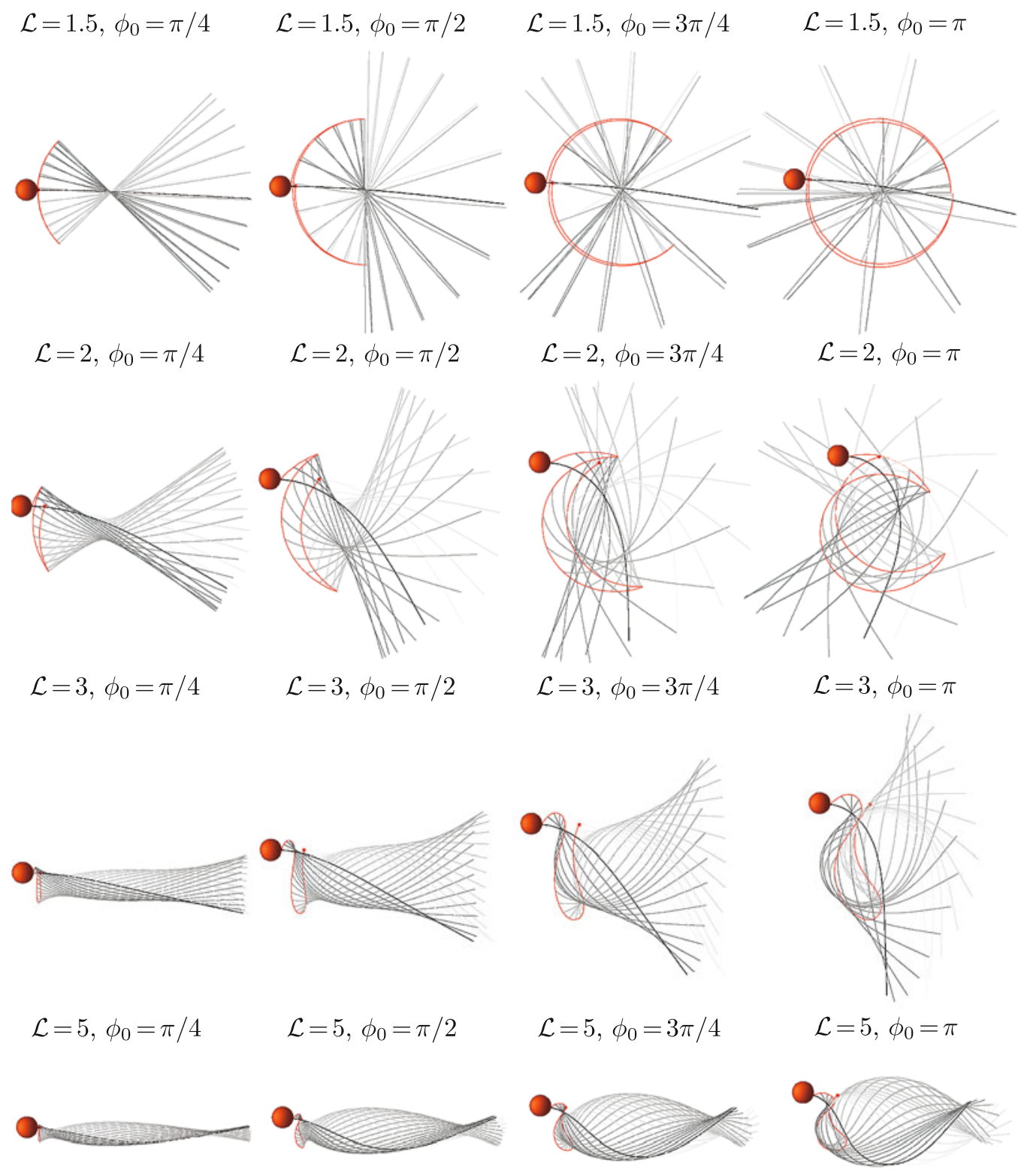

Fig. 2. (Color online) Swimming behavior of a spherical head magnetic swimmer with $\ell=1$, and magnetic number $m=50$, for $\mathcal{L} \in[1.5,5]$ and $\phi_{0}=\pi / 4, \pi / 2,3 \pi / 4, \pi$, as indicated. The evolution of the flexible tail is plotted at equal time intervals over one period (darker curves for later times), while the trajectory of the head-tail junction at $s=0$ is depicted by the red curves.

while keeping $|\hat{\boldsymbol{h}}(t)|=1$, with an angular amplitude of oscillation $\phi_{0}$. This particular choice of magnetic actuation describes magnetic fields with a constant time-averaged orientation, directed to $-\boldsymbol{x}$, which is ultimately responsible for defining the swimming direction of the magnetic device. Finally, periodicity is expected from the imposed magnetic field in Eq. (3.1), and therefore the time iteration is continued until consecutive solutions, one period apart, are identical.

We begin by contrasting the swimming behaviors for two distinct head geometries in Figs. 2 and 3 , for the case of a simple spherical magnetic head, $\ell=1$, and an elongated prolate spheroidal head, $\ell=6$, respectively. For each magnetic head swimmer, the tail-compliance parameter varied from effectively stiff, $\mathcal{L}=1.5$, to effectively flexible filaments, $\mathcal{L}=5$, while the imposed angular amplitude of the magnetic field $\phi_{0}$ varied between $\pi / 4$ and $\pi$. The most evident feature in both Figs. 2 and 3 is the emergence of a wide range swimming patterns with distinctive complexity, as both $\mathcal{L}$ and $\phi_{0}$ are augmented. The head-junction trajectories, depicted by the red curves, ranges from circular, cusp-like shapes for $\mathcal{L}=1.5,2$ to smooth curvature patterns for $\mathcal{L}=3,5$. The area 

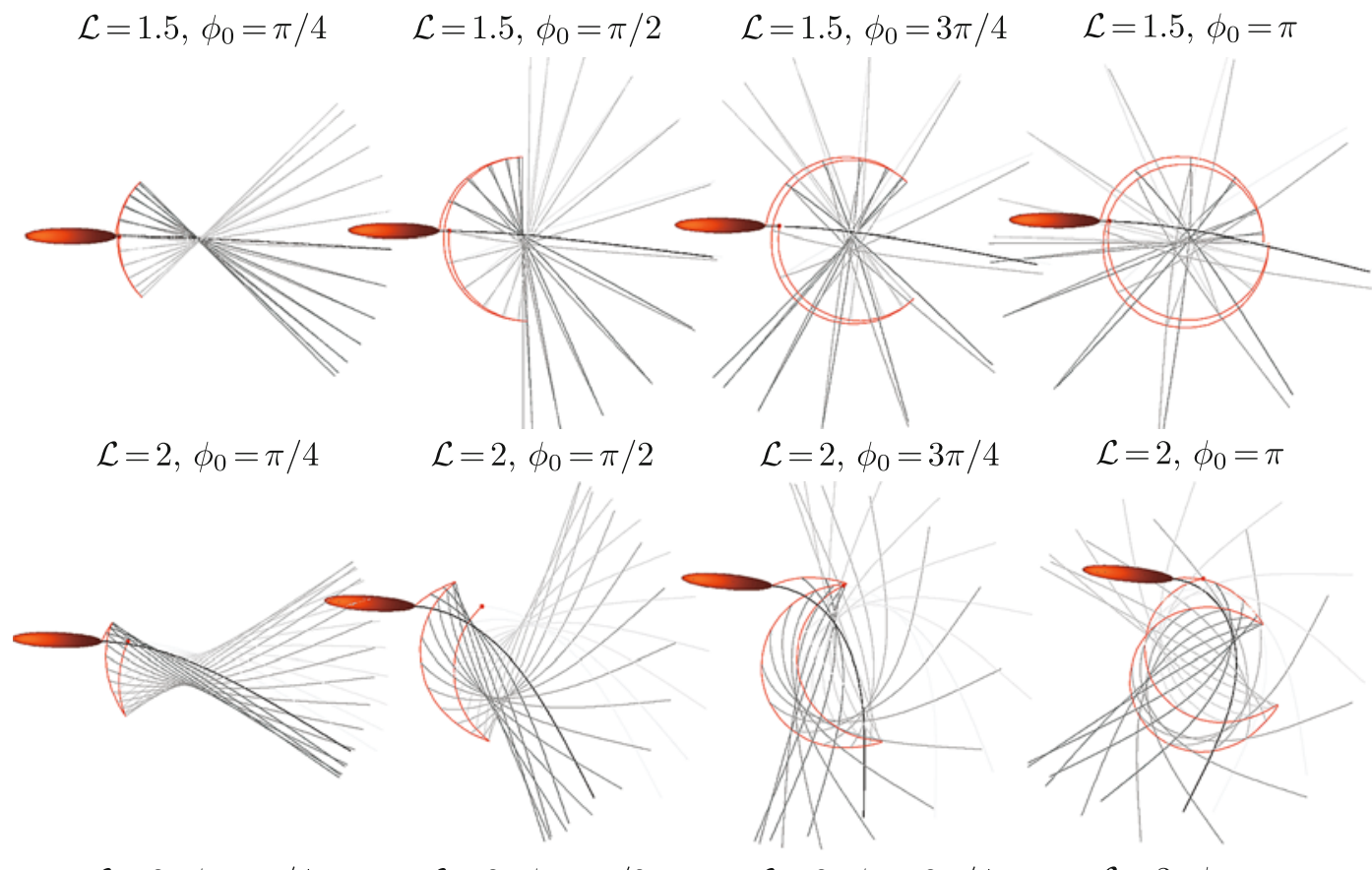

$\mathcal{L}=3, \phi_{0}=\pi / 4 \quad \mathcal{L}=3, \phi_{0}=\pi / 2 \quad \mathcal{L}=3, \phi_{0}=3 \pi / 4$
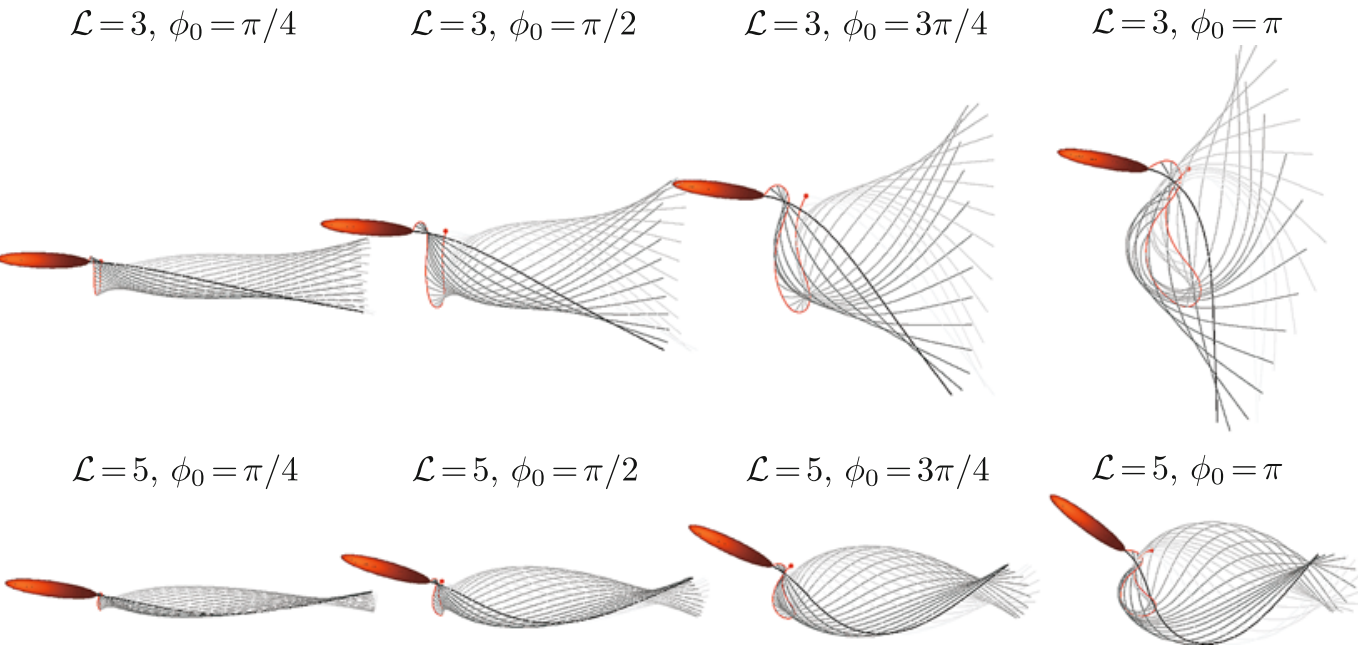

Fig. 3. (Color online) Swimming behavior of a magnetic swimmer with an elongated prolate spheroidal head, $\ell=6$, and magnetic number $m=50$, for $\mathcal{L} \in[1.5,5]$ and $\phi_{0}=\pi / 4, \pi / 2,3 \pi / 4, \pi$, as indicated. The evolution of the flexible tail is plotted at equal time intervals over one period (darker curves for later times), while the trajectory of the head-tail junction at $s=0$ is depicted by the red curves.

covered by the flexible tail over a period is equally modified by the pair $\left(\mathcal{L}, \phi_{0}\right)$. As one would expect, by increasing the angular amplitude of the magnetic field, the angular variation of the magnetic head trajectory is equally increased. Nevertheless, the latter is still regulated by the tailcompliance parameter, which tends to dampen the imposed magnetic actuation for larger values of $\mathcal{L}$, corresponding to an increasing viscous domination of the dynamics or, alternatively, a higher frequency of oscillation; compare, for instance, $\mathcal{L}=1$ to 5 for $\phi_{0}=\pi$ in Fig. 2. The resulting propulsion is, however, an intricate consequence of the flexible tail covering a fluid volume, or project area for slender filaments, while minimizing the low Reynolds number time-reversibility constraint, Purcell's scallop theorem [25], by allowing elastic bending deformations. This explains, for instance, the almost non-existent net propulsion observed for effectively stiff tails, $\mathcal{L}=1.5$ in Figs. 2 and 3 despite the large projected area covered by the elastic filament $[29,30]$.

Now we turn our attention to the influence of the magnetic field on the total travelled distance $d$ performed by the magnetic swimmer over one period. Fig. 4 plots $d$ as a function of the tailcompliance parameter $\mathcal{L}$ for the spherical and prolate spheroidal head geometries with the same 

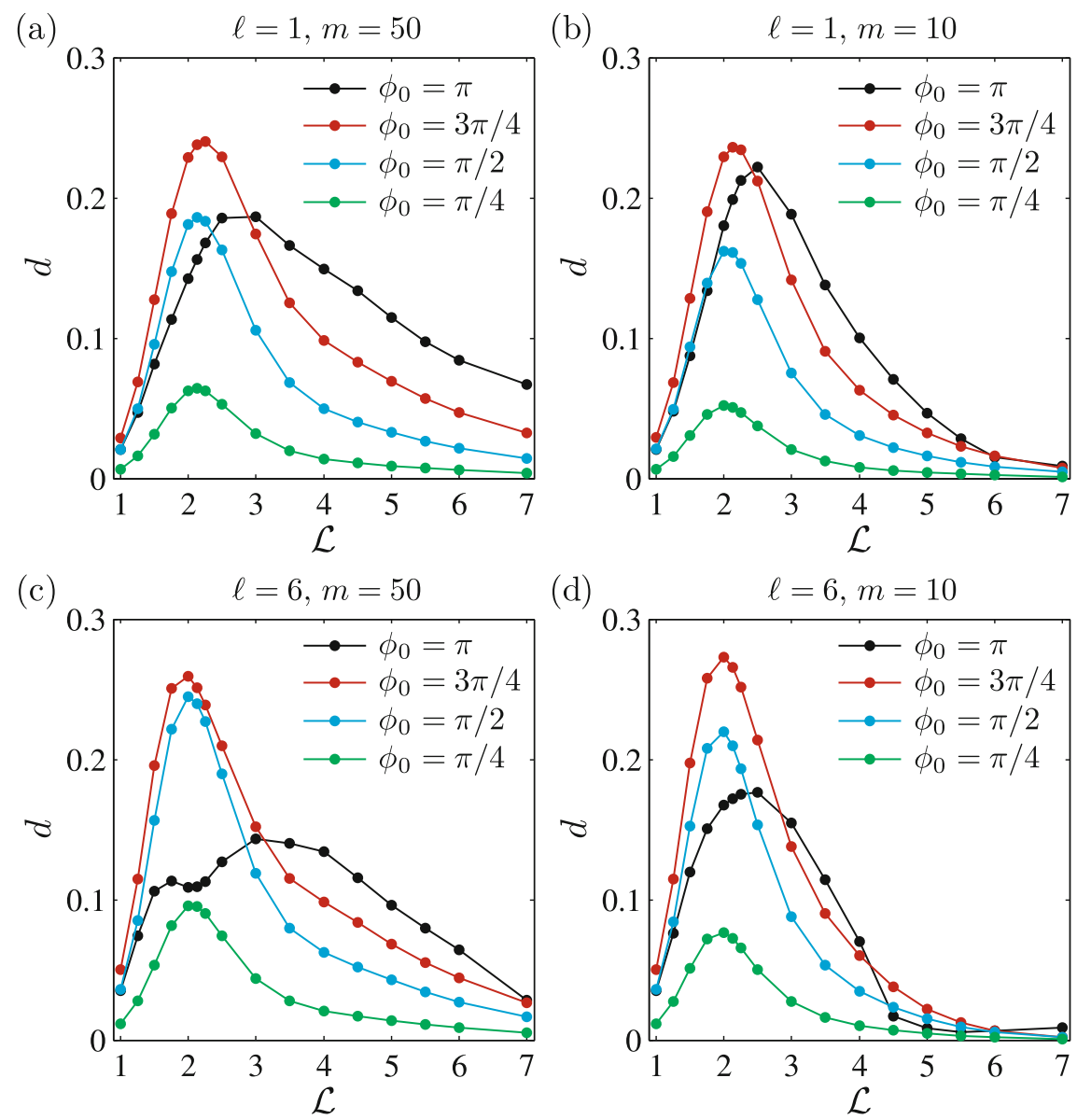

Fig. 4. (Color online) Total swimming distance $d$, over one period of oscillation, as a function of the tailcompliance parameter $\mathcal{L}$ for four distinct angular amplitudes $\phi_{0}$, as indicated by the colored lines. Results for the spherical (prolate spheroidal) head are shown in the top (bottom) rows for two different magnetic numbers $m$.

angular amplitude of the imposed magnetic field used in Figs. 2 and 3. As one would expect from the elastohydrodynamic formulation of pivoting elastic filaments $[29,30]$, the net propulsion is characterized by a maximum, typically around $\mathcal{L} \approx 2.2$. The maximum value of the swimming distance, $d_{\max }$, however, is a non-monotonic function of the amplitude $\phi_{0}$ and is represented by the formation of a global maximum around $\phi_{0}=3 \pi / 4$ for any given head geometry and magnetic number, cf. Fig. 4. Furthermore, Fig. 4 shows how changes in the magnetic number have little effect in $d$ for effectively stiff filaments, that is when $\mathcal{L}$ is low. The opposite scenario is found for the viscous dominated regime $\mathcal{L}>4$, where $d$ is marked by a rapid decay with $\mathcal{L}$, when the magnetic number is weak.

The central result from this elastohydrodynamic formulation is depicted in Fig. 5, where we contrast the hydrodynamic effect of the head geometry on the traveled distance $d$ of the magnetic swimmer for six different $\left(m, \phi_{0}\right)$-pairs, with $\ell \in[1,6]$. Despite the similarities between the tail beating associated with different head geometries in Figs. 2 and 3, the head shape plays an important role in regulating the total swimming distance. With the exception of Figs. $5, c, f$ for the extreme case $\phi_{0}=\pi$, continuous elongation of the head shape acts to increase $d_{\text {max }}$ for any pair $\left(m, \phi_{0}\right)$. Nevertheless, after reaching the maximum, the total swimming distance is observed to have a faster decay with $\mathcal{L}$ for larger values of the slenderness parameter $\ell$, if the magnetic number is sufficiently weak (see, e.g., Figs. $5, b, d$ and $e$ ). The head shape hydrodynamics have therefore opposing effects depending on the tail-compliance parameter. For the elastic dominated regime, when $\mathcal{L}$ is low, the magnetic swimmer is marked by a large head yawing, Fig. 2, which is reduced by the non-symmetric viscous friction arising from the elongated head geometry, working to ultimately favor the forward motion. For the viscous dominated case, the effective hydrodynamic 

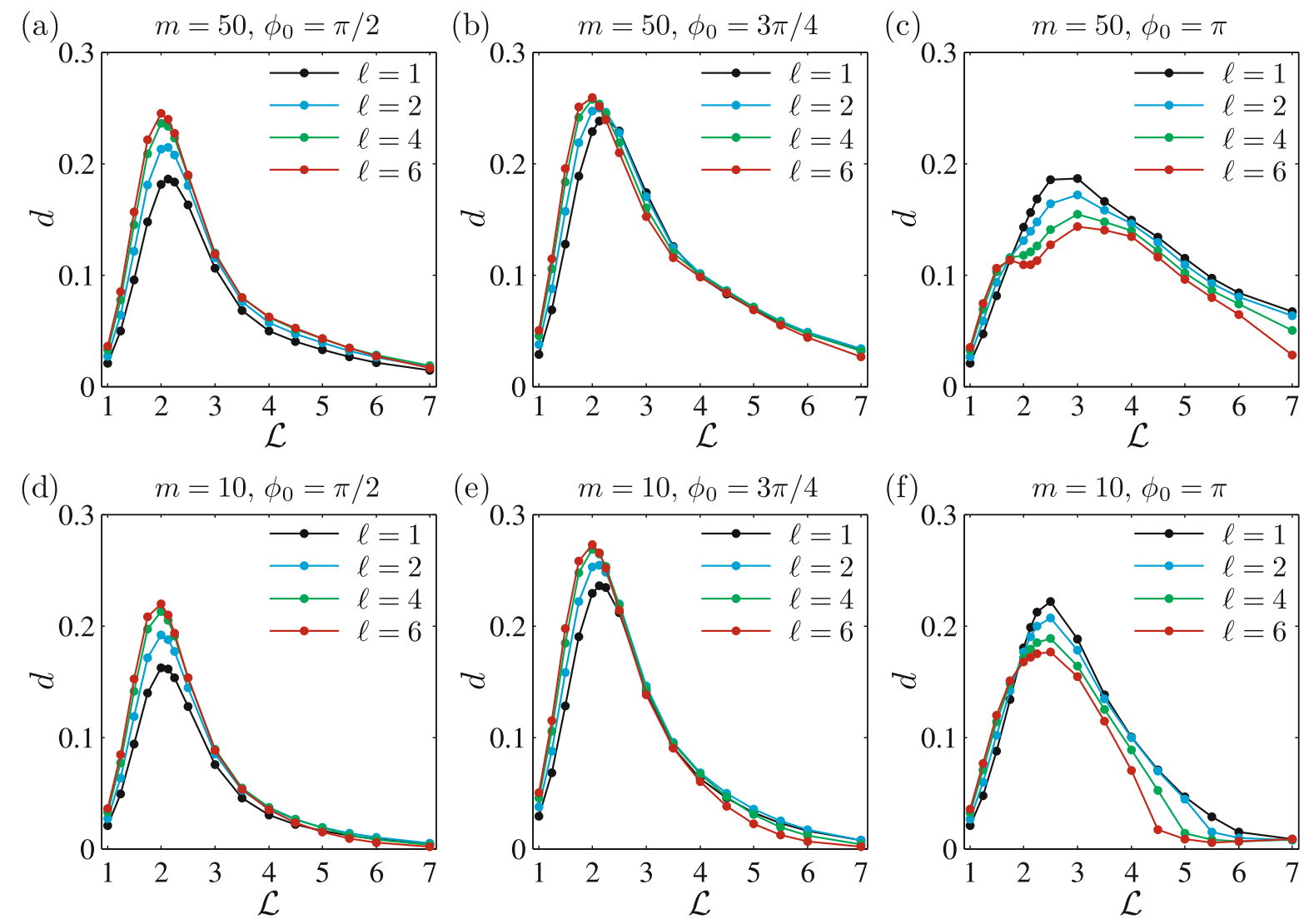

Fig. 5. (Color online) Total swimming distance $d$, over one period of oscillation, as a function of the tailcompliance parameter $\mathcal{L}$ for four distinct head geometries $\ell$, as indicated by the colored lines. Results for high (low) magnetic number are shown in the top (bottom) rows for three different angular amplitudes of the magnetic field $\phi_{0}$.

drag experienced by the head is already sizable and even augmented if the head aspect ratio increases, cf. Fig. 3, reducing in this way the potential for forward propulsion. The decrease in the swimming speed for large $\mathcal{L}$, however, is still influenced by $\phi_{0}$ and $m$, which can be chosen accordingly to circumvent the large viscous head drag (see, e.g., Fig. 5, c). Recall that we are contrasting head geometries with the same volume. Surprisingly enough, the overall maximum in $d$ was found when the magnetic number is low, as shown in Fig. 5, $e$ for $\ell=6$, with an angular amplitude $\phi_{0}=3 \pi / 4$ as expected from the behavior displayed in Fig. 4 . If the magnetic number is large, the magnetic swimmer rigidly follows the angular oscillation imposed by the magnetic field, regardless of the tail configuration. The angle $\alpha$ between the magnetic moment and the imposed magnetic field reaches a maximum value in time of only $\alpha=0.0298 \pi$ for a strong magnetic field, $m=50$, while for a low magnetic number, $m=10$, the phase lag between the head orientation and the oscillatory magnetic field can be five times larger, $\alpha=0.1473 \pi$, for the optimum case shown in Fig. 5, $e$ with $\ell=6$ and $\mathcal{L}=2$. Due to the hydrodynamic friction, the delay between the orientation of the magnetic moment and the imposed magnetic field is used constructively to favor propulsion by allowing the stored elastic energy from the deformed tail to relax during the phase lag of the magnetic moment, when the head is reorienting to the magnetic field at the same time as the large head aspect ratio directs the swimmer into a forward motion, as discussed above. In particular, this result suggests the existence of optimal magnetic actuations that are non-smooth, and even discontinuous in time, in order to take full advantage of the propulsive potential associated with the relaxation dynamics of the deformed elastic tail.

A large head aspect ratio is not always beneficial for the net propulsion of the swimmer, as demonstrated in Fig. 5, $c, f$. When the angular amplitude of the magnetic field is large, elongated head geometries induce an increasingly high viscous friction due to the long time spent with the head orientation directed away from, and perpendicular to, the direction of propulsion. The latter is minimized by using a spherical head when $\phi_{0}$ is large. In particular, an even better performance for 
this regime may be achieved by considering oblate spheroids, or by connecting instead the elastic tail in such a way that the filament tangent vector coincides with the prolate spheroid's minor axis. It is also noteworthy that although $\phi_{0}=\pi$ reduces $d_{\max }$, Fig. 4 , a slower decay of the travelled distance with $\mathcal{L}$ is observed, see Fig. 5, c. Hence, the swimming speed is considerably larger for the viscous dominated regime if the angular amplitude is $\pi$, contrast, for example, the values of $d$ for $\ell=1, \mathcal{L}=7$ in Fig. 5, $a, b$ with Fig. 5, c. Furthermore, Fig. 5 shows that the head aspect ratio $\ell$ modifies not only the maximum speed of the swimmer, but also, for a given magnetic number $m$ and angular amplitude $\phi_{0}$, the location of the maximum $\mathcal{L}_{\text {max }}$. While in Figs. 5, $a$ and $d \mathcal{L}_{\text {max }}$ remains unchanged when $\ell$ is varied, $\mathcal{L}_{\max }$ tends to decrease with $\ell$ for the cases shown in Figs. 5, $b, e$ and $f$. The opposite scenario is depicted in Fig. $5, c$, which shows $\mathcal{L}_{\text {max }}$ increasing with $\ell$, in addition to the formation of a local minimum at the same tail-compliance that maximizes $d$ for $\phi_{0} \leqslant 3 \pi / 4$, see Fig. 4, $c$ for clarity.

\section{CONCLUSIONS}

Although the performance of artificial magnetic swimmers with a flexible tail has been discussed in detail, the influence of the body geometry on the swimming speed and ultimate shape of the beating patterns has been markedly overlooked. In this work we tackled the problem by employing an elastohydrodynamic formulation for the flexible tail, while coupling the intrinsic hydrodynamic forces and torques associated with the prolate spheroidal magnetic head, together with the imposed moments from oscillatory magnetic field. The resulting geometrically non-linear equations were solved numerically for a wide range of head aspect ratios and distinct magnetic field configurations.

Our geometrically non-linear formulation revealed a wide range of swimming patterns with increased complexity, depending on the tail-compliance parameter $\mathcal{L}$ and the angular amplitude of the magnetic field $\phi_{0}$, as illustrated in Figs. 2 and 3. Despite the similarities between the beating patterns associated with different head geometries, the swimming speed is dramatically modified by the head aspect ratio. In general, the added asymmetric hydrodynamic moments from elongated prolate spheroids act to suppress the non-forward motion due to the head yawing, favoring in this way the net propulsion of the magnetic swimmer. The latter, in particular, is in agreement with the geometrically linear formulation of non-magnetic swimmers that are driven, instead, by pivoting an elastic tail [21]. Nevertheless, for a large head aspect ratio, the swimming speed, averaged over a period, is found to be maximized for $\mathcal{L} \approx 2$, when the magnetic number $m$ is low and $\phi_{0}=3 \pi / 4$, see Fig. $5, e$. In this case, smaller values of the magnetic number allow viscous friction to ultimately induce a time delay between the orientation of the imposed magnetic field and the swimmer's magnetic moment, which is directed along the spheroid's polar axis, so that the head orientation does not coincide with the instantaneous direction of the external magnetic field. Because of this weak magnetic coupling, the artificial swimmer is able to constructively use the time-delayed head orientation, in the swimming direction, by releasing the stored elastic energy from the deformed tail in order to promote a larger forward motion. This further exemplifies that large magnetic numbers are not necessary in order to maximize the swimming speed of magnetic swimmers. Furthermore, this result suggests the possibility of maximizing the swimming speed by considering an even richer space of non-smooth and discontinuous periodic functions for the magnetic actuation, with the objective to take advantage of the relaxation dynamics of the elastic tail.

We have also found that the swimming speed can be maximized by considering the appropriate angular amplitude of the magnetic field, Fig. 4. The maximum net propulsion is in fact a nonmonotonic function of $\phi_{0}$, characterized by the formation of a maximum around $\phi_{0}=3 \pi / 4$, as illustrated in Fig. 4. Nevertheless, despite the distinctive role of the angular amplitude, the swimmer's performance can be improved by tuning the appropriate head parameter with the magnetic field strength. This is illustrated in Fig. 5, $c, f$, where the large head aspect ratio is in fact reducing the maximum speed, so that the spherical head geometry offers a superior performance. Furthermore, because of the rapid decay of the swimming distance $d$ with tail-compliance parameter, for $\mathcal{L}>\mathcal{L}_{\max }$, characteristic of elastohydrodynamic systems [21, 29-31], a large angular amplitude of the magnetic field and large magnetic numbers are required to achieve a sizeable forward motion in the viscous dominated regime.

Finally, the numerical investigation presented here showed the possibility of engineering not only the maximum swimming speed of the magnetic device, $d_{\max }$, but also the location of the maximum in the tail-compliance parameter space, $\mathcal{L}_{\max }$, by considering non-linear interactions from the magnetic head geometry and the nature of the imposed magnetic field via the triplet $\left(\ell, \phi_{0}, m\right)$. 
Additionally, our geometrically non-linear formulation can be equally employed to consider a higher class of mathematical problems in optimal control theory, applied to achieve, for instance, a better maneuverability of the magnetic swimmer, or to minimize instead the transient state from the above non-linear dynamical system. A systematic search in the space of parameters, and periodic functions for the magnetic actuation, may also provide valuable insight into the physical limit of the swimming speed of elasto-magnetic devices. In summary, we hope our current work will stimulate further investigations on the role of oscillatory magnetic fields and the body shape geometry during the design of artificial magnetic devices.

\section{ACKNOWLEDGMENTS}

We are indebted to Eamonn A. Gaffney for many helpful discussions and continued insight. H.G. acknowledges the WYNG Foundation and Trinity Hall, University of Cambridge, for financial support.

\section{REFERENCES}

1. Abbott, J. J., Peyer, K. E., Lagomarsino, M. C., Zhang, L., Dong, L., Kaliakatsos, I. K., and Nelson, B. J., How Should Microrobots Swim?, Int. J. Robot. Res., 2009, vol. 28, pp. 1434-1447.

2. Antman, S. S., Nonlinear Problems of Elasticity, 2nd ed., Appl. Math. Sci., vol. 107, New York: Springer, 2005.

3. Belovs, M. and Cebers, A., Ferromagnetic Microswimmer, Phys. Rev. E, 2009, vol. 79, 051503, 5 pp.

4. Coq, N., Ngo, S., du Roure, O., Fermigier, M., and Bartolo, D., Three-Dimensional Beating of Magnetic Microrods, Phys. Rev. E, 2010, vol. 82, 041503, 10 pp.

5. Dreyfus, R., Baudry, J., Roper, M. L., Fermigier, M., Stone, H. A., and Bibette, J., Microscopic Artificial Swimmers, Nature, 2005, vol. 437, pp. 862-865.

6. Erglis, K., Livanovics, R., and Cebers, A., Three Dimensional Dynamics of Ferromagnetic Swimmer, J. Magn. Magn. Mater., 2011, vol.323, no. 10, pp.1278-1282.

7. Gadêlha, H., Gaffney, E. A., Smith, D. J., and Kirkman-Brown, J. C., Nonlinear Instability in Flagellar Dynamics: A Novel Modulation Mechanism in Sperm Migration?, J. R. Soc. Interface, 2010, vol.7, pp. 1689-1697.

8. Gaffney, E. A., Gadêlha, H., Smith, D. J., Blake, J.R., and Kirkman-Brown, J. C., Mammalian Sperm Motility: Observation and Theory, Annu. Rev. Fluid Mech., 2011, vol.43, pp. 501-528.

9. Gao, W., Kagan, D., Pak, O.Sh., Clawson, C., Campuzano, S., Chuluun-Erdene, E., Shipton, E., Fullerton, E. E., Zhang, L., Lauga, E., and Wang, J., Cargo-Towing Fuel-Free Magnetic Nanoswimmers for Targeted Drug Delivery, Small, 2012, vol. 8, no.3, pp. 460-467.

10. Gauger, E., Downton, M., and Stark, H., Fluid Transport at Low Reynolds Number with Magnetically Actuated Artificial Cilia, Eur. Phys. J. E., 2009, vol. 28, no. 2, pp. 231-242.

11. Goldstein, R. E. and Langer, S. A., Nonlinear Dynamics of Stiff Polymers, Phys. Rev. Lett., 1995, vol. 75, pp. 1094-1097.

12. Gray, J. and Hancock, G. J., The Propulsion of Sea-Urchin Spermatozoa, J. Exp. Biol., 1955, vol.32, pp. 802-814.

13. Honda, T., Arai, K. I., and Ishiyama, K., Micro Swimming Mechanisms Propelled by External Magnetic Fields, IEEE Trans. Magn., 1996, vol. 32, no. 5, pp. 5085-5087.

14. Ishiyama, K., Sendoh, M., Yamazaki, A., and Arai, K. I., Swimming Micro-Machine Driven by Magnetic Torque, Sensor. Actuat. A Phys., 2001, vol. 91, nos. 1-2, pp. 141-144.

15. Jackson, J. D., Classical Electrodynamics, 3rd ed., New York: Wiley \& Sons, 1999.

16. Kantsler, V. and Goldstein, R.E., Fluctuations, Dynamics, and the Stretch-Coil Transition of Single Actin Filaments in Extensional Flows, Phys. Rev. Lett., 2012, vol. 108, 038103, 5 pp.

17. Keaveny, E. E. and Maxey, M.R., Spiral Swimming of an Artificial Micro-Swimmer, J. Fluid Mech., 2008, vol. 598, pp. 293-319.

18. Khaderi, S. N., Baltussen, M. G. H. M., Anderson, P. D., Ioan, D., den Toonder, J. M. J., and Onck, P. R., Nature-Inspired Microfluidic Propulsion Using Magnetic Actuation, Phys. Rev. E, 2009, vol. 79, 046304, $4 \mathrm{pp}$.

19. Kim, S. and Karrila, S. J., Microhydrodynamics: Principles and Selected Applications, New York: Dover, 2005.

20. Landau, L.D., Pitaevskii, L.P., Lifshitz, E.M., and Kosevich, A.M., Theory of Elasticity, 3rd ed., Oxford: Butterworth-Heinemann, 1986.

21. Lauga, E., Floppy Swimming: Viscous Locomotion of Actuated Elastica, Phys. Rev. E, 2007, vol.75, 041916, $16 \mathrm{pp}$.

22. Livanovics, R. and Cebers, A., Magnetic Dipole with a Flexible Tail As a Self-Propelling Microdevice, Phys. Rev. E, 2012, vol. 85, 041502, 5 pp.

23. Machin, K. E., Wave Propagation along Flagella, J. Exp. Biol., 1958, vol. 35, pp. 796-806. 
24. Pak, O.Sh., Gao, W., Wang, J., and Lauga, E., High-Speed Propulsion of Flexible Nanowire Motors: Theory and Experiments, Soft Matter, 2011, vol. 7, no. 18, pp. 8169-8181.

25. Purcell, E. M., Life at Low Reynolds Number, Amer. J. Phys., 1977, vol. 45, pp. 3-11.

26. Roper, M., Dreyfus, R., Baudry, J., Fermigier, M., Bibette, J., and Stone, H. A., On the Dynamics of Magnetically Driven Elastic Filaments, J. Fluid Mech., 2006, vol. 554, pp. 167-190.

27. Sudo, S., Segawa, S., and Honda, T., Magnetic Swimming Mechanism in a Viscous Liquid, J. Intel. Mat. Syst. Str., 2006, vol. 17, nos. 8-9, pp. 729-736.

28. Tierno, P., Golestanian, R., Pagonabarraga, I., and Sagues, F., Controlled Swimming in Confined Fluids of Magnetically Actuated Colloidal Rotors, Phys. Rev. Lett., 2008, vol. 101, 218304, 4 pp.

29. Wiggins, C.H. and Goldstein, R.E., Flexive and Propulsive Dynamics of Elastica at Low Reynolds Number, Phys. Rev. Lett., 1998, vol. 80, 3879-3882.

30. Wiggins, C. H., Riveline, D., Ott, A., and Goldstein, R. E., Trapping and Wiggling: Elastohydrodynamics of Driven Microfilaments, Biophys. J., 1998, vol. 74, pp. 1043-1060.

31. Yu, T. S., Lauga, E., and Hosoi, A.E., Experimental Investigations of Elastic Tail Propulsion at Low Reynolds Number, Phys. Fluids, 2006, vol. 18, 0917011, 4 pp. 